\title{
First report of tobacco rattle virus in hydrangea in China
}

\author{
${\text { Zhao-Lin } \mathrm{Ji}^{2} \text { • Peng-Xiang Zhu }}^{2} \cdot$ Gen-He He ${ }^{1}$ Y Yong-Hui Liao ${ }^{1} \cdot$ Feng $\mathrm{Zhu}^{2}$ \\ Received: 28 July 2019 / Accepted: 20 August 2019 / Published online: 4 September 2019 \\ (C) Società Italiana di Patologia Vegetale (S.I.Pa.V.) 2019
}

Keywords Tobacco rattle virus $\cdot$ Hydrangea macrophylla $\cdot$ China

In July 2018, foliar virus-like symptoms such as stunting and mottling were observed in plants of Hydrangea macrophylla in Yangzhou City, Jiangsu Province, east China. Leaf samples were collected from two symptomatic hydrangea plants and assayed by Western blotting using polyclonal antisera to cucumber mosaic virus (CMV), and tobacco mosaic virus (TMV) (Zhu et al. 2013) as well as by double-antibody sandwich (DAS)-ELISA for tobacco rattle virus (TRV). Results revealed the two samples were positive for TRV. To further confirm the presence of TRV in hydrangea, reverse transcription (RT)-PCR was performed using total RNA extracted from leaves with Trizol reagent (Invitrogen, Carlsbad, CA, USA). The first-strand cDNA was prepared using M-MLV Reverse Transcriptase (Invitrogen). PCR was performed using primers identical to nucleotides 1923 to 1938 (TRV1-F: 5'-ATCC GTGCCGCTATTA-3') and complementary to nucleotides 2320 to 2335 (TRV1-R: 5'-TGTACCGCTTGTTTCC-3'), which were designed to amplify a partial region of the RNA1-encoded replicase gene (GenBank accession No. AF406990). The expected 413 bp amplicon was amplified from the two symptomatic leaf samples that were positive for TRV in DAS-ELISA. The obtained products were purified

Yong-Hui Liao

liaoyonghui@jgsu.edu.cn

Feng Zhu

zhufeng@yzu.edu.cn

1 School of Life Sciences, Key Laboratory of Biology diversity and Ecological Engineering of Jiangxi Province, Jinggangshan University, Ji'an 343009, China

2 College of Horticulture and Plant Protection, Yangzhou University, Yangzhou 225009, Jiangsu, China by TIANgel Midi Purification Kit (Tiangen, Beijing, China), cloned into the pMD19-T vector (TaKaRa, Dalian, China) and sequenced. The 413 bp consensus sequence named TRV-JS09 was deposited in GenBank under accession number MK795824. BLASTN analysis revealed that the TRS-JS-09 sequence had $98-100 \%$ identity to eight TRV sequences available in GenBank (KY817142, AF166084, AF406990, AF314165, KT033406, KT033407, D00155 and AF034622). To our knowledge, this is the first report of TRV in hydrangea in China.

Acknowledgements This study was supported by the Qing Lan Project of Yangzhou University and the earmarked fund for Modern Agroindustry Technology Research System (CARS-30-3-02).

\section{Compliance with ethical standards}

Conflict of interest The authors declare that they have no conflict of interest.

Research involving human participants and/or animals This article does not contain any studies with human participants or animals performed by any of the authors.

Informed consent This manuscript is new and not being considered elsewhere. All authors have approved the submission of this manuscript.

\section{Reference}

Zhu F, Zhang P, Meng YF, Xu F, Zhang DW, Cheng J, Lin HH, Xi DH (2013) Alpha-momorcharin, a RIP produced by bitter melon, enhances defense response in tobacco plants against diverse plant viruses and shows antifungal activity in vitro. Planta 237:77-88

Publisher's note Springer Nature remains neutral with regard to jurisdictional claims in published maps and institutional affiliations. 